\title{
Nonlinear magnetoelectric effects in flexible composite ferromagnetic - piezopolymer structures
}

\author{
L.Y.Fetisov ${ }^{1}$, I.A. Baraban ${ }^{2}$, Y.K. Fetisov ${ }^{1}$, D.A.Burdin ${ }^{1}$, M.M.Vopson ${ }^{3}$ \\ ${ }^{1}$ Moscow Technological University (MIREA), 119454, Russia \\ ${ }^{2}$ Immanuel Kant Baltic Federal University, Russia \\ ${ }^{3}$ University of Portsmouth, Faculty of Science, SEES, Portsmouth, UK
}

\begin{abstract}
Nonlinear magnetoelectric (ME) effects in a flexible composite planar structure, containing mechanically coupled layers of amorphous $\mathrm{FeBSiC}$ ferromagnet and PVDF piezoelectric polymer have been experimentally investigated. Under the action of a weak harmonic magnetic field $h$ with the frequency $f=50-1000 \mathrm{~Hz}$ and tangential bias magnetic field $H=1-80 \mathrm{Oe}$, the structure generated a voltage of the same frequency. The efficiency of a linear ME conversion reached $3.4 \mathrm{~V} /(\mathrm{cm} \cdot \mathrm{Oe})$ for the optimum bias field $H_{\mathrm{m}} \approx 15$ Oe. On increasing the excitation field up to $h \sim 7 \mathrm{Oe}$, the structure generated the second and the third harmonics with efficiencies of $\sim 25 \mathrm{mV} /\left(\mathrm{cm} \cdot \mathrm{Oe}^{2}\right)$ and $\sim 2.5 \mathrm{mV} /\left(\mathrm{cm} \cdot \mathrm{Oe}^{3}\right)$, respectively. The amplitudes of the harmonics were not monotonous functions on the bias field $H$ and grew with the increase in the alternating field $h$. Under the action of two alternating fields with different frequencies $f_{1}$ and $f_{2}$, the structure generated ac voltages with frequencies equal to the sum and difference frequencies $f_{1} \pm f_{2}$. The efficiency of magnetic fields mixing reached a maximum of $\sim 30$ $\mathrm{mV} /\left(\mathrm{cm} \cdot \mathrm{Oe}^{2}\right)$ in the absence of the bias field. The effects of harmonics generation and magnetic fields mixing arise due to the nonlinear dependence of the ferromagnet's magnetostriction $\lambda$ on the bias field $H$. The efficiency of the nonlinear processes is proportional to the derivatives of the magnetostriction over magnetic field. The nonlinear ME effects in the ferromagnet-piezopolymer flexible structures can be used to design high-sensitivity dual ac / dc magnetic field sensors and energy harvesting devices.
\end{abstract}

\section{Introduction}

Magnetoelectric (ME) effect in composite materials exhibits itself as an electrical polarization of a sample in an external magnetic field (direct effect) or a change of magnetization of the sample in an external electric field (converse effect) [1]. The effect is observed in singlephase materials and in composite materials which simultaneously posses magnetic and electric ordering [2]. The largest direct $\mathrm{ME}$ effect was observed in planar structures containing alternating ferromagnetic (FM) and piezoelectric (PE) layers. The ME effect in such structures arises due to a combination of magnetostriction of the FM layer and piezoelectricity of the PE layer through mechanical coupling between the layers [3]. The magnitude of the direct effect is characterized by the magnetically induced $\mathrm{ME}$ voltage coefficient $\alpha_{\mathrm{E}}=\delta E / \delta H$, where $\delta E$ is the amplitude of the electric field generated by the magnetic field $\delta H$.

It was shown $[2,4]$, that in structures containing FM layers with high magnetostriction $\lambda$ (metals Ni, Co, alloys FeCo, FeGa, Terfenol-D, amorphous alloys, ferrites) and PE layers with large piezomodulus $d$ (ceramic lead zirconate titanate (PZT), lead magnesium niobate - lead titanate crystals, AlN, langatate, quartz) the ME coefficient can reach values of $\alpha_{\mathrm{E}} \sim 1-10$ $\mathrm{V} /(\mathrm{cm} \cdot \mathrm{Oe})$. The effect can be enhanced by up to two orders of magnitude when the excitation field frequency coincides with the frequency of acoustic resonance of the structure [5], which is defined by the size and stiffness of the material used. These resonance frequencies for bulk samples are typically in the range from a few $\mathrm{kHz}$ to hundreds of $\mathrm{kHz}$. Promising applications of the ME effect in composite structures are: highly sensitive magnetic field sensors, wireless energy harvesting devices, new data recording and storage elements, and electrically tuned radiofrequency signal processing devices [4, 6-8].

In recent years, the interest in the study of ME effects in composite structures comprising piezopolymers, especially polyvinilidene fluoride (PVDF), has increased significantly [9-11] because of a number of advantages that PVDF has over ceramic and single crystal piezoelectric materials $[11,12]$. In particular, the PVDF fabrication technology is well developed and, unlike 
the ceramic piezoelectrics, it does not require high temperature processing. Although the PVDF piezomodulus is not very large, $d_{31} \approx 6-20 \mathrm{pC} / \mathrm{N}$, this is compensated by its low permittivity $\varepsilon$ $\approx 12$, resulting in a ratio $d_{31} / \varepsilon \sim 1$, which is several times higher than that of most crystals and piezoceramics. The polymer has a high resistivity $\left(\rho>10^{14} \mathrm{Om} \cdot \mathrm{cm}\right)$ and small dielectric losses $(\operatorname{tg} \delta$ $\approx 0.02$ ). The combination of all these properties imply the expectation of high values of the ME coefficient for the structures consisting of PVDF layers. In addition, the Young's modulus of the PVDF, $Y \approx 3 \mathrm{GPa}$, is an order of magnitude smaller than that of the single crystals and piezoelectric ceramics, i.e. it is usually referred to as "soft" material. This makes PVDF ideal for the use in the low frequency range $\sim 1-10^{3} \mathrm{~Hz}$ applications, which coincide with the frequencies of natural and industrial vibrations as well as magnetic field frequencies. Moreover, the PVDF can be used to fabricate flexible structures that can be deposited onto surfaces of complex forms, it has a significantly lower cost in comparison with crystalline and ceramic piezoelectrics and is biocompatible in some cases, which is important for medical applications.

The ME effect was first observed in the planar structure of Terfenol-D-PVDF [9], in structures with PVDF layers of the following compositions: PZT/Terfenol-D/PVDF [13], NiMn-Ga-PVDF [14], PVDF-Vitrovac [15-17], and in structures with nanometer-thick layers of Fe-PVDF-Fe [18]. Flexible structures exhibiting ME effects were first made by bonding PVDF and amorphous Metglas layers [19], resulting in non resonance ME coefficients of $\alpha_{E}=7.2$ $\mathrm{V} /(\mathrm{cm} \cdot \mathrm{Oe})$ and $\alpha_{\mathrm{E}} \approx 238 \mathrm{~V} /(\mathrm{cm} \cdot \mathrm{Oe})$ at the resonance frequency of $50 \mathrm{kHz}$. In subsequent works [20-23] it was shown that Metglas is the most suitable material for fabrication of flexible ME structures with PVDF layers, because it has high magnetostriction coefficient $\lambda \sim 20-30 \mathrm{ppm}$, it saturates in low magnetic fields $H_{\mathrm{S}} \sim 50 \mathrm{Oe}$, and PVDF layers can be deposited directly on the ferromagnetic layer [24] without using glue. This allowed to achieve, under resonance conditions, the maximum ME coefficient of $\alpha_{\mathrm{E}}=850 \mathrm{~V} /(\mathrm{cm} \cdot \mathrm{Oe})$ in structures containing PVDF layers. Based on such structures, magnetic field sensors with limit of detection as small as $~ 1 \mathrm{pT}$ [25], current sensors [26], and the energy harvesters [27] have been demonstrated.

It should be noted that till now the ME effects in structures with piezopolymer layers were investigated only for small amplitudes of excitation magnetic fields and the signal generated by the structure was registered at the frequency of excitation magnetic field. From physical point of view and for various applications the study of nonlinear ME effects in the polymerbased structures is of great interest. Such effects may be observed at relatively large amplitudes of the fields due to nonlinear dependence of the magnetostriction of a ferromagnetic layer on the magnetic field [28-29].

In the present paper we report, for the first time, experimental studies of the nonlinear ME effects in composite layered FM-PE structures, consisting of piezoelectric polymer PVDF and amorphous alloy Metglas. We show that, for two ac magnetic field excitations applied simultaneously, the PVDF layer effectively generates a voltage of multiple frequencies with values equal to the difference and sum of the excitation frequencies, especially when the amplitudes of alternating magnetic fields acting on the structure are comparable or larger than the dc bias field. The first part of the paper describes the structure under investigation and the experimental setup, followed by the results of experimental investigation of linear and nonlinear ME effects in the Metglas-PVDF structure, discussion of the results and conclusions.

\section{Samples and experimental setup}

Fig. 1a shows a schematic view of the structure under investigation, while Fig. 1b shows its appearance. The ferromagnetic layer was made of amorphous iron-based ribbon FeBSiC (Metglas Inc.). It has lateral dimensions of $10 \mathrm{~mm}$ x $14 \mathrm{~mm}$ and thickness of $27 \mu \mathrm{m}$. The choice of Metglas is due to its high saturation magnetostriction $\lambda_{\mathrm{s}} \approx 20 \cdot 10^{-6}$, sufficiently small saturation field $H_{\mathrm{S}} \sim 50 \mathrm{Oe}$, and good mechanical properties, including high flexibility. The piezoelectric layer was made of a commercially available PVDF film (TE Connectivity Ltd.). 
It had lateral dimensions of $10 \mathrm{~mm} \times 14 \mathrm{~mm}$ and the thickness of $b=28 \mu \mathrm{m}$.

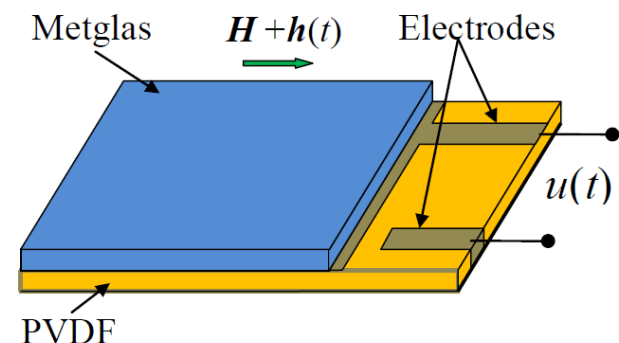

(a)

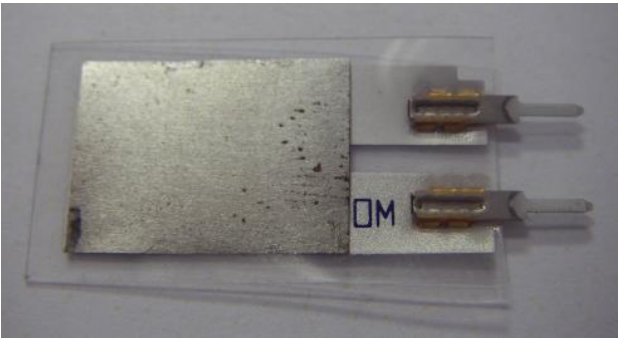

(b)

Fig. 1 Schematic view (a) and appearance (b) of the Metglas-PVDF structure.

$3 \mu \mathrm{m}$ thick Ag electrodes were deposited on the surfaces of piezoelectric layer. The PVDF layer had capacitance $\mathrm{C}=0.55 \mathrm{nF}$ measured at $1 \mathrm{kHz}$ frequency, which corresponds to the dielectric permittivity $\varepsilon=11.6$. The piezoelectric modulus of the PVDF was $d_{31} \approx 10 \mathrm{pC} / \mathrm{N}$. The Metglas and PVDF layers were mechanically coupled under the press using the fast dry "Loctite" adhesive.

The block-diagram of the experimental setup is shown in Fig. 2. The structure was placed in a uniform bias dc magnetic field $H=0-100$ Oe created by a pair of Helmholtz coils, which were fed dc current from the "DC power supply" AKIP 1125. Alternating magnetic fields $h_{1} \cos \left(2 \pi f_{1} t\right)$ and $h_{2} \cos \left(2 \pi f_{2} t\right)$ with amplitudes $h_{1}, h_{2}$ up to 7 Oe and frequencies in the range $f_{1}, f_{2}$ $=10 \mathrm{~Hz}-5 \mathrm{kHz}$ were created by two coaxial electromagnetic coils $\mathrm{K}_{1}$ and $\mathrm{K}_{2}$, which were powered by two independent generators "AC Gen1" and "AC Gen2", both Agilent 33210A. Magnetic fields were measured using "Gaussmeter" LakeShore 421 with accuracy of 0.1 Oe. The ME signal was amplified using an "Amp" SR 560 amplifier and registered using an "Oscilloscope" TDS 3032A. The amplitude of generated ac voltage was measured using the "Voltmeter" AKIP2401.

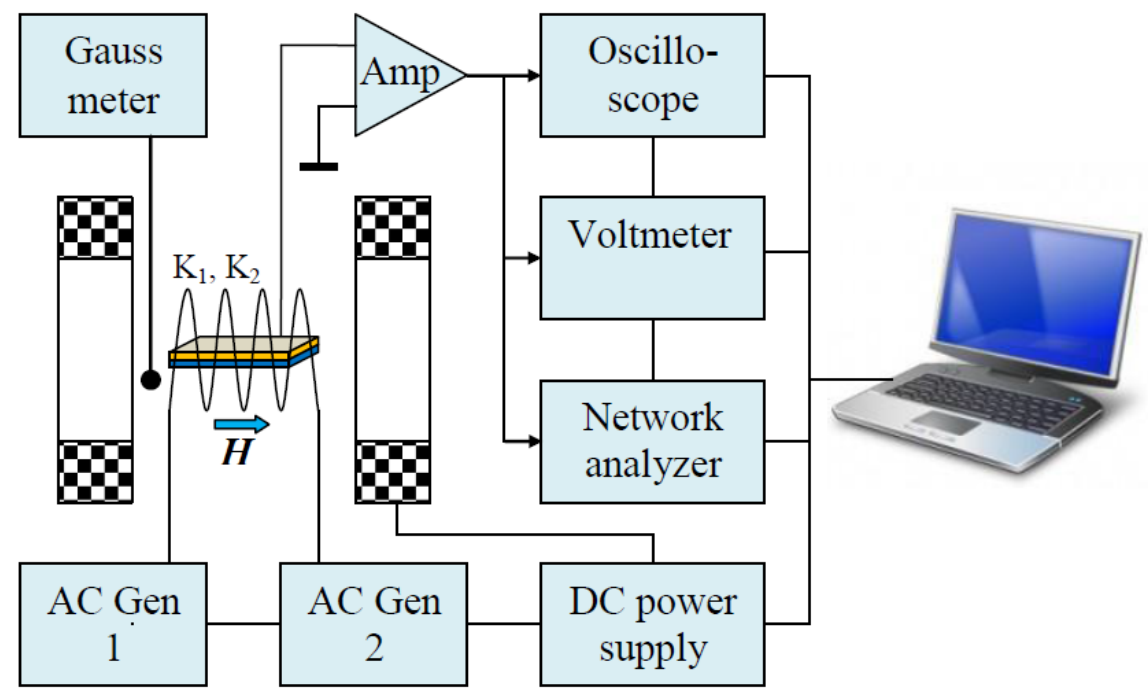

Fig. 2 Block-diagram of the experimental setup. 
The frequency spectra of the output signal were registered by the "Network Analyzer" SR770. The entire experiment and data processing were fully automated and controlled using a special LabView program.

\section{Experimental results}

At the first stage, we have measured the frequency and field characteristics of the direct ME effect in the described structure under its excitation in linear regime with a small amplitude ac magnetic field. Figure 3a shows frequency dependence of the voltage $u(f)$ generated by the PVDF layer under excitation with magnetic field amplitude $h=1$ Oe and bias field $H=15$ Oe. The structure generates voltage in the frequency range from $\sim 50 \mathrm{~Hz}$ up to $\sim 5 \mathrm{kHz}$. At low frequencies $f<100 \mathrm{~Hz}$ the voltage decreases due to finite conductivity of the PE layer [30], and at higher frequencies $f>2 \mathrm{kHz}$ the voltage decreases due to lowering in the amplitude of excitation magnetic field caused by the inductance of the coils. The low-Q peak near the frequency $\sim 1.2$ $\mathrm{kHz}$ with the quality factor $Q \approx 5$ occurs, as it was shown by the formulas assessment [31], due to the resonance excitation of bending oscillations of the structure. The maximum value of the
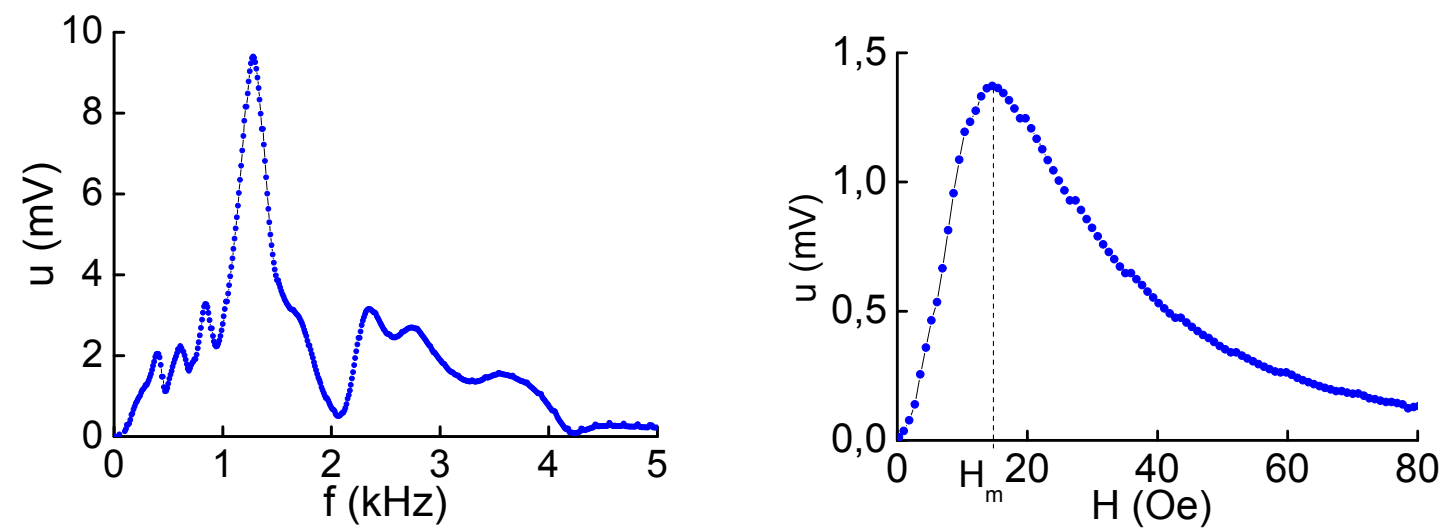

Fig. 3 (a) Amplitude-frequency characteristic of the ME effect at $h=1$ Oe and $H=15$ Oe,

(b) Dependence of the ME voltage vs. bias field $H$ at $h=1$ Oe and $f=500 \mathrm{~Hz}$.

ME coefficient at resonance frequency reached $\alpha_{\mathrm{E}} \approx 3.4 \mathrm{~V} /(\mathrm{cm} \cdot \mathrm{Oe})$. At low frequencies $\sim 200 \mathrm{~Hz}$ - $1 \mathrm{kHz}$, where the amplitude-frequency response of the ME effect was more uniform, the ME coefficient was $\alpha_{\mathrm{E}} \approx 0.3-1.2 \mathrm{~V} /(\mathrm{cm} \cdot \mathrm{Oe})$. The below described studies of non resonant nonlinear ME effects were carried out in this frequency range.

Figure $3 \mathrm{~b}$ shows the magnetic field dependence of the generated voltage $u(H)$ when the structure was excited by ac field $h=1$ Oe with a frequency $f=500 \mathrm{~Hz}$. The response is typically characteristic for planar structures [2]: the voltage first grows almost linearly with increasing of $H$, reaches a maximum at $H_{\mathrm{m}} \approx 15 \mathrm{Oe}$, which corresponds to the maximum of piezomagnetic coefficient $\lambda^{(1)}(H)=\partial \lambda / \partial H$ of the ferromagnetic layer, and then gradually decreases with increasing $H$ during the saturation of the magnetostriction. From data presented in Fig. 3, the ME coefficient was estimated as $\alpha_{E}=u /(b h) \approx 0.47 \mathrm{~V} /(\mathrm{cm} \cdot \mathrm{Oe})$ at optimum bias field $H_{\mathrm{m}}$.

At the second stage the characteristics of ME effect in the Metglas-PVDF structure have been measured in the spectral region under single-frequency excitation for various values of ac and dc magnetic fields. Typical results of the measurements are shown in Fig. 4.

Figure 4a shows the normalized voltage waveform generated by the PVDF layer of the structure when it is excited by ac magnetic field of low amplitude $h=1$ Oe with frequency $f=$ $200 \mathrm{~Hz}$ at bias field $H=15$ Oe. Figure $4 \mathrm{~b}$ shows the corresponding voltage spectrum. The spec- 
trum contains only one component at a frequency equal to the frequency of the excitation field $f$ $=200 \mathrm{~Hz}$. Using data presented in Fig.4, the value of linear ME coupling coefficient was estimated as $\alpha_{E}^{(1)}=u_{1} /(b h) \approx 0.46 \mathrm{~V} /(\mathrm{cm} \cdot \mathrm{Oe})$.

Figure $4 \mathrm{c}$ and Figure $4 \mathrm{~d}$ show the normalized waveform and corresponding voltage frequency spectrum, generated by the PVDF layer under excitation in the nonlinear regime with ac magnetic field of large amplitude $h=6$ Oe at the same frequency $f=200 \mathrm{~Hz}$ and for bias field $H$ $=4$ Oe. It is seen that the waveform is greatly distorted, while the second and the third voltage harmonics with frequencies of $400 \mathrm{~Hz}$ and $600 \mathrm{~Hz}$ appeared in the spectrum.
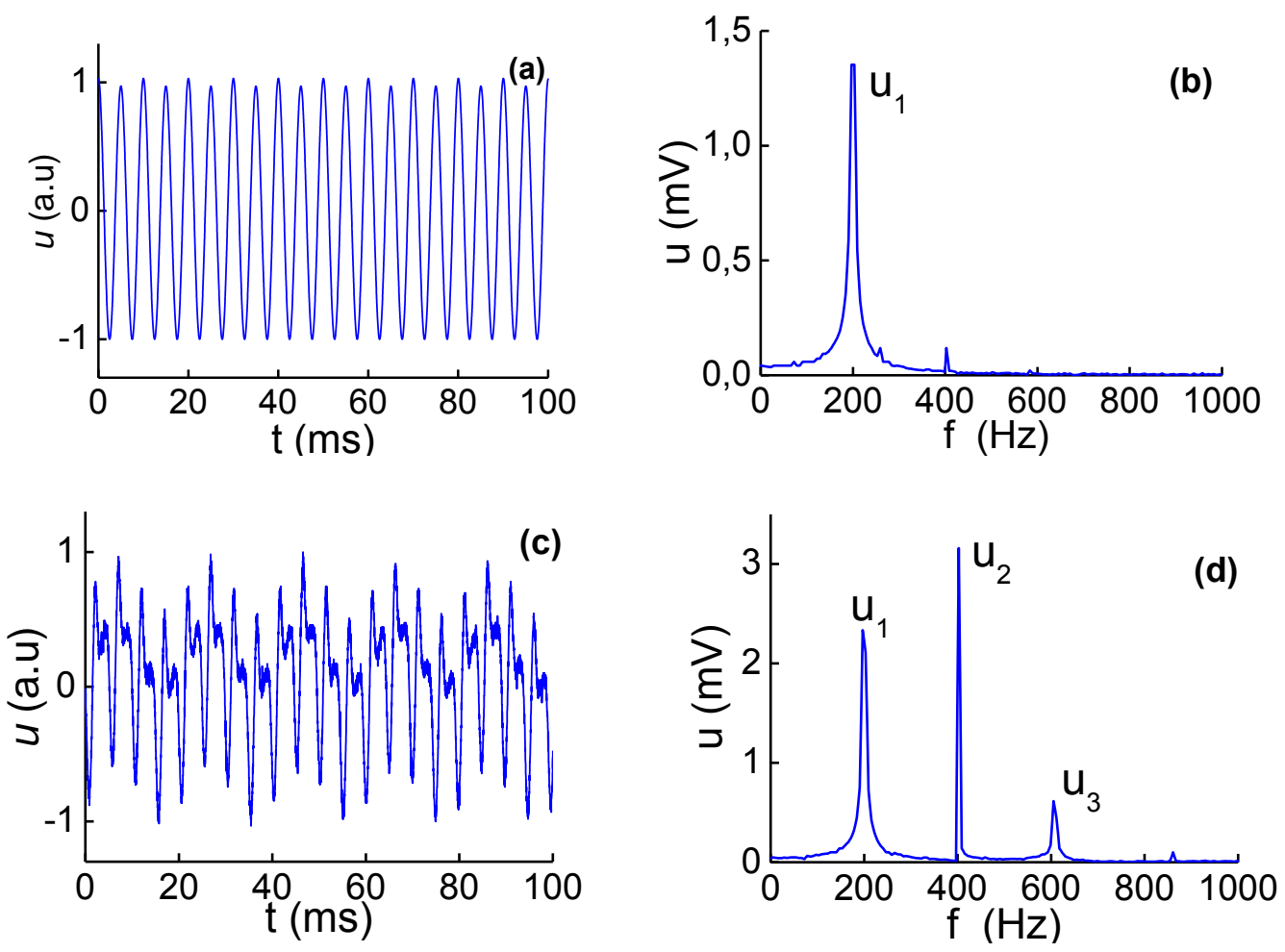

Fig. 4 a) The waveform (a) and frequency spectrum (b) of the voltage, generated by the PVDF-Metglas structure in the linear regime at $h=1$ Oe and $H=15$ Oe; the waveform (c) and frequency spectrum (d) of the voltage, generated by the ME structure in the nonlinear regime at $h=6$ Oe and $H=4$ Oe.

Amplitude of the second harmonic is larger by a factor of $\sim 1.35$ than the amplitude of the first harmonic, and by a factor of $\sim 5$ than the amplitude of the third harmonic. The coefficients of ME conversion for these harmonics for the given frequency and fields were $\alpha_{E}^{(1)}=u_{1} /(b h) \approx 140$ $\mathrm{mV} /(\mathrm{cm} \cdot \mathrm{Oe}), \alpha_{E}^{(2)}=u_{2} /\left(b h^{2}\right) \approx 30 \mathrm{mV} /\left(\mathrm{cm} \cdot \mathrm{Oe}^{2}\right)$, and $\alpha_{E}^{(3)}=u_{3} /\left(b h^{3}\right) \approx 1 \mathrm{mV} /\left(\mathrm{cm} \cdot \mathrm{Oe}^{3}\right)$, respectively.

Figure 5 shows measured dependencies of the harmonic amplitudes vs. bias field $H$ at the excitation field $h=4$ Oe and frequency $f=200 \mathrm{~Hz}$. It is seen that the amplitude of the first harmonic reaches maximum $u_{1} \approx 5.5 \mathrm{mV}$ at $\sim 16$ Oe field, which is slightly larger than the field corresponding to the maximum of the ME voltage in the linear regime. The amplitude of the second harmonic in zero field is $u_{2} \approx 0.8 \mathrm{mV}$, with a maximum $\sim 1.2 \mathrm{mV}$ at the $\sim 7$ Oe field and a second maximum at $\sim 22$ Oe field. The third harmonic also has two maxima with amplitudes less than $0.3 \mathrm{mV}$ in fields of $\sim 2$ Oe and $\sim 20$ Oe. 


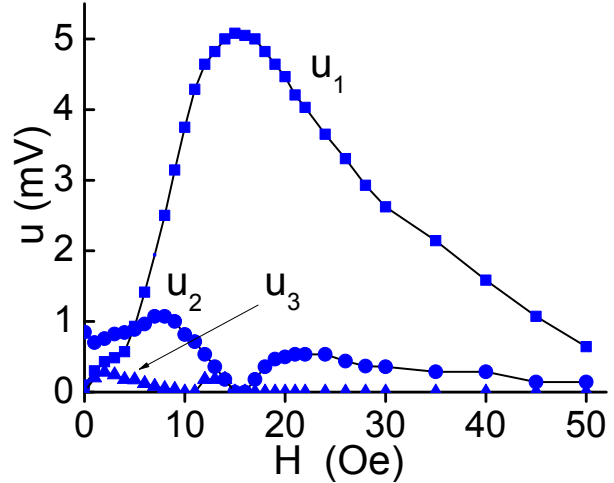

Fig. 5 DC magnetic field dependences of the first $u_{1}$, second $u_{2}$ and the third $u_{3}$ harmonics of the ME voltage for $h=4$ Oe and $f=200 \mathrm{~Hz}$.

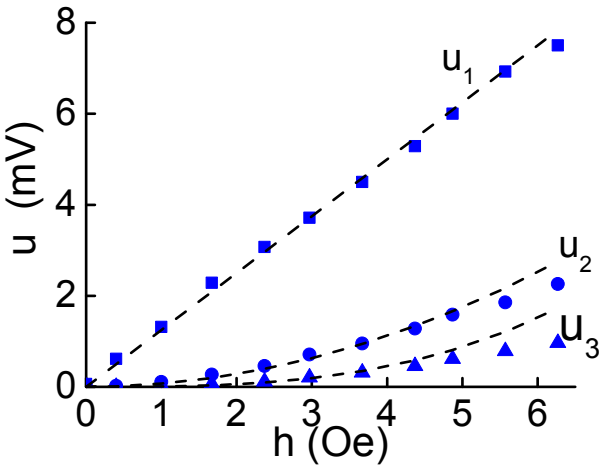

Fig. 6 AC magnetic field dependences of the first $u_{1}$ at $H=16 \mathrm{Oe}$, second $u_{2}$ at $H=7$ Oe and the third $u_{3}$ at $H=2$ Oe harmonics. Dashed lines are the approximations of experimental data by power functions.

Figure 6 shows measured dependences of the harmonic amplitudes vs. excitation field $h$ at constant fields $H$, corresponding to the maximum for each harmonics in Fig. 5.

The dashed lines show approximations for each dependence by power functions. It can be seen that the results are well described by the theory in the excitation field range $h<4$ Oe, which will be discussed shortly.

At the third stage we investigated nonlinear mixing of ac magnetic fields in the PVDFMetglas structure. In order to do this, the structure was excited with two ac magnetic fields of frequencies $200 \mathrm{~Hz}$ and $300 \mathrm{~Hz}$ and amplitudes $h_{1}=h_{2}=4$ Oe. The voltage response frequency spectrum of the PVDF was registered.

Figure 7a shows frequency spectrum of ME voltage when the structure is excited by ac field with frequency $200 \mathrm{~Hz}$ and $h=6 \mathrm{Oe}$ at $H=0$. Figure $7 \mathrm{~b}$ shows frequency spectrum when the structure is excited by ac magnetic field with frequency $300 \mathrm{~Hz}$ and $h=6 \mathrm{Oe}$, also at $H=0$. In both figures the additional second harmonics are seen at frequencies $400 \mathrm{~Hz}$ and $600 \mathrm{~Hz}$, respectively. Figure 7c shows the ME voltage frequency spectrum when the structure is excited by two ac magnetic fields. In addition to the peaks of the first and second harmonics with frequencies 200, 300, 400, and $600 \mathrm{~Hz}$, one can notice peaks at frequencies $100 \mathrm{~Hz}$ and $500 \mathrm{~Hz}$. These peaks correspond to magnetic fields mixing and are described by the formula:

$$
f_{ \pm}=f_{1} \pm f_{2} .
$$

Amplitudes of the peaks were approximately equal to each other and reached a value of $\sim 3.3 \mathrm{mV}$. They reached maximum at $H \approx 0$ and changed qualitatively in the same way at the increase in $\mathrm{H}$, similar to the amplitude of the second voltage harmonic $u_{2}(H)$ in Fig. 5.

Figure 8 shows measured amplitude of the peak with sum frequency $u\left(f_{+}\right)$, generated by the Metglas-PVDF structure as a function of the pumping field amplitudes. The data was acquired by fixing the amplitude of the field $h_{2}$, while amplitude of the field $h_{1}$ was varied in the range 0 - 7 Oe. and amplitude of the peak with sum frequency was registered. It is seen that the peak signal grows with an increase of the excitation field $\mathrm{h}_{1}$. Similar dependencies at nonlinear field mixing conditions were obtained for the peak with difference frequency $u\left(f_{-}\right)$. In both cases the maximum efficiency of nonlinear mixing was $\alpha_{E}^{m i x}=u\left(f_{ \pm}\right) /\left(b h_{1} h_{2}\right) \approx 30 \mathrm{mV} /\left(\mathrm{cm} \cdot \mathrm{Oe}^{2}\right)$. Similar measurements were carried out for other frequencies $f_{1}$ and $f_{2}$ of excitation magnetic fields in the range of $50-1000 \mathrm{~Hz}$. It was found that for frequency difference $\left|f_{2}-f_{1}\right|<500 \mathrm{~Hz}$ the effi- 
ciency of ac fields mixing $\alpha_{E}^{m i x}$ was almost constant and changed only because of irregularity of the voltage frequency characteristic (see Fig. 2)

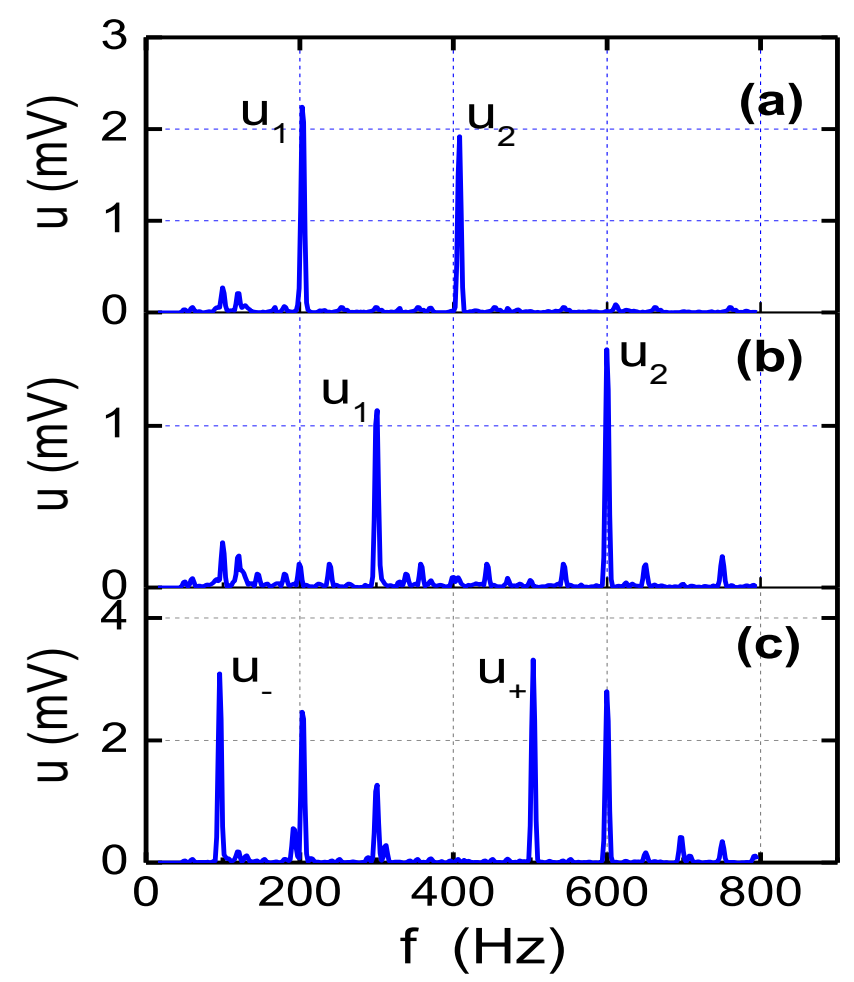

Fig. 7 Demonstration of the ac magnetic field mixing in the Metglas - PVDF structure: a) frequency spectrum of ME voltage for the structure excitation with ac field $f_{1}=200 \mathrm{~Hz}$;

b) frequency spectrum of $\mathrm{ME}$ voltage for the structure excitation with ac field $f_{2}=300 \mathrm{~Hz}$;

c) frequency spectrum of ME voltage for the structure excitation with two ac fields of frequencies $f_{1}$ and $f_{2}$.

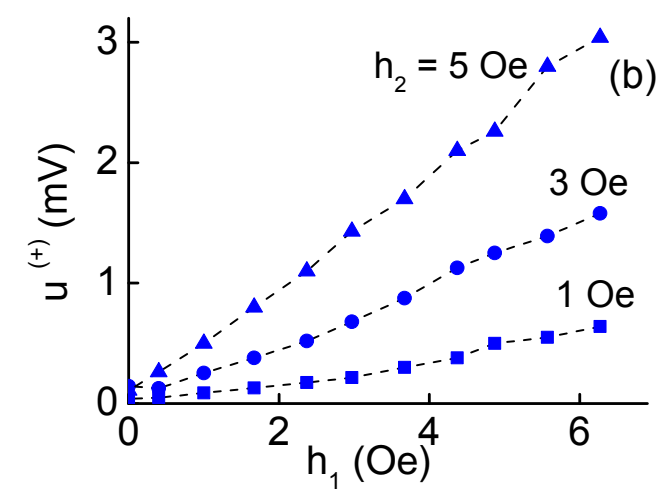

Fig.8 Dependences of the voltage with sum frequency $u_{+}\left(f_{1}+f_{2}\right)$ on the amplitude of the excitation field $h_{1}$ under magnetic field mixing conditions in the PVDF-Metglas structure for $h_{2}=1 \mathrm{Oe}, 3 \mathrm{Oe}$, and $5 \mathrm{Oe}$. 


\section{Discussion of results}

In order to explain the experimental results we use the approach developed in [32. 33????]. The composite structure comprising of mechanically coupled FM and PE layers placed in a dc bias magnetic field $H$, due to the combination of magnetostriction and piezoeffect generates a voltage $u=A d_{31} \lambda(H)$, where $A$ is the constant coefficient depending only on the size of the layers and their electrical and magnetic parameters. When exposed to the harmonic excitation field $H(t)=H+h \cos (2 \pi f \mathrm{t})$ (where $\left.h \ll H_{\mathrm{S}}\right)$ the structure generates an alternating voltage: $u(t)=$ $u_{1} \cos (2 \pi f t)+u_{2} \cos (4 \pi f t)+u_{3} \cos (6 \pi f t)+\ldots$. Expanding the magnetostriction $\lambda(H)$ in a Taylor series and retaining only the highest terms of the expansion, we get the following expressions for the harmonic amplitudes:

$$
\begin{aligned}
& u_{1}=A d \lambda^{(1)} h+\frac{1}{8} A d \lambda^{(3)} h^{3} \approx A d \lambda^{(1)} h, \text { the first harmonic. } \\
& u_{2} \approx \frac{1}{4} A d \lambda^{(2)} h^{2}, \text { the second harmonic. } \\
& u_{3} \approx \frac{1}{24} A d \lambda^{(3)} h^{3}, \text { the third harmonic. }
\end{aligned}
$$

Coefficients $\lambda^{(1)}=\partial \lambda / \partial H, \lambda^{(2)}=\partial^{2} \lambda / \partial H^{2}$, and $\lambda^{(3)}=\partial^{3} \lambda / \partial H^{3}$ in (2) are the derivatives of magnetostriction $\lambda$ in respect with the field $H$ at fixed bias field???

Thus, the nonlinear dependence of the magnetostriction of the ferromagnetic phase on the dc bias field should lead to the generation of the voltage harmonics, that was observed during the experiment. The dependence of the harmonic amplitude $u_{\mathrm{n}}$ on $H$, according to the theory, is determined by the shape of the field dependence of the corresponding derivatives $\lambda^{(\mathrm{n})}$. Figure $9 \mathrm{a}$ shows the dependence of the Metglas layer deformation vs. dc magnetic field, measured via the strain gauge method. Figures $9 b-d$ show the dependencies of the first three derivatives of

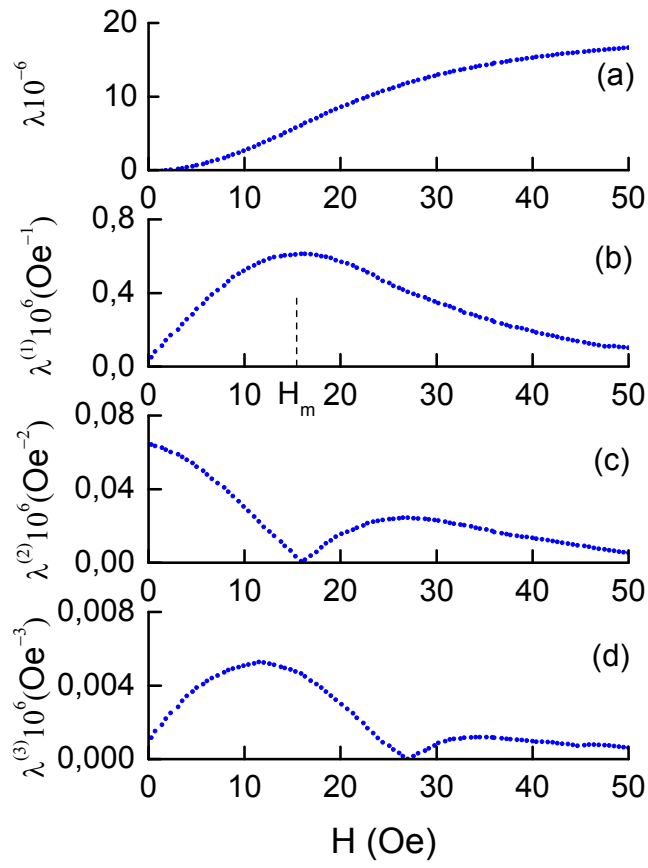

Fig. 9 Magnetostriction curve $\lambda(H)$ for the Metglas layer (a) and derivatives of the magnetostriction over the field $H: \lambda^{(1)}(\mathrm{b}), \lambda^{(2)}$ (c), and $\lambda^{(3)}(\mathrm{d})$. magnetostriction vs. magnetic field, numerically calculated using the data of Fig. 9a. It is seen, that maximum values of the second and the third derivatives are approximately one and two orders of magnitude, respectively, lower than the first derivative.

The efficiency of the generation of the second and the third voltage harmonics should be smaller or the same orders as for derivatives. Comparison of the curves in Fig. 5 and fig. 9 shows good qualitatively agreement between experimental data and theoretical predictions. It can be seen, that amplitude of the first harmonic $u_{1}$ and the derivative $\lambda^{(1)}$ increase linearly with $H$ near zero value and have one maximum at the optimum bias field $H_{\mathrm{m}} \approx 15 \mathrm{Oe}$. The amplitude of the second harmonic $u_{2}$ and the value of the derivative $\lambda^{(2)}$ reach maximum in the fields $H$ $=0-6 \mathrm{Oe}$, after that they fall to zero in the field region $\sim 15$ Oe and have one more maximum at higher fields. The amplitude of the third harmonic $u_{3}$ and the third derivative $\lambda^{(3)}$ grow linearly with increasing $H$ in weak 
fields range and have an additional maximum at higher fields. It also follows from (2) that amplitudes of the harmonics are the power functions of the excitation field $u_{\mathrm{n}} \sim h^{\mathrm{n}}$. Calculated approximating curves $u_{1}=1.25 h, u_{2}=0.17 h^{2}$ and $u_{3}=0.007 h^{3}$ are represented in Fig. 6 as a proof. One can see a good qualitative match between data and theoretical predictions in the range of the excitation fields $h<4$ Oe. If follows from data shown in Fig. 6 that the harmonics generation efficiencies are: $\alpha_{E}^{(1)}=u_{1} /(b h) \approx 0.45 \quad \mathrm{~V}(\mathrm{~cm} \cdot \mathrm{Oe}), \quad \alpha_{E}^{(2)}=u_{23} /\left(b h^{2}\right) \approx 25 \mathrm{mV} /\left(\mathrm{cm} \cdot \mathrm{Oe}^{2}\right), \quad$ and $\alpha_{E}^{(3)}=u_{3} /\left(b h^{3}\right) \approx 2.5 \mathrm{mV} /\left(\mathrm{cm} \cdot \mathrm{Oe}^{3}\right)$, respectively. The approach used (i.e. expansion of $\lambda(H)$ into a Taylor series at $h \ll H_{\mathrm{S}}$ ) becomes inapplicable with an increase of the excitation field $h$, which explains the discrepancy between experimental data and calculated curves for excitation fields with high amplitudes $h>4$ Oe.

It should be noted, that described dependence of the harmonics amplitudes on the dc bias field $H$ and ac excitation field $h$ can lead to the fact that amplitudes of the second and the third harmonic can exceed the amplitude of the main harmonic for a certain values of $H$ and $h$, as it was observed experimentally (see Fig. 4d).

When the structure exposed to the two-frequency excitation field $H(t)=H+$ $h_{1} \cos \left(2 \pi f_{1} t\right)+h_{2} \cos \left(2 \pi f_{2} t\right)$, similarly we get that structure should generate spectral components of the voltage with the sum and difference frequencies:

$$
u(t)=u_{-} \cos \left[2 \pi\left(f_{1}-f_{2}\right) t\right]+u_{+} \cos \left[2 \pi\left(f_{1}+f_{2}\right) t\right]
$$

The amplitudes of the components are equal to each other and and given by the formula:

$$
u_{-}=u_{+}=\frac{1}{4} A d_{31} \lambda^{(2)} h_{1} h_{2}
$$

It follows from (4), that amplitudes of these components are proportional to the second derivative of the magnetostriction $\lambda^{(2)}$, i.e. should have maximum at $H=0$ and decrease to zero by increasing the bias field up to $H_{\mathrm{m}} \approx 15 \mathrm{Oe}$. This exactly dependence of the amplitudes of the components was observed in the experiment. Furthermore, the amplitudes of the components should increase linearly with increasing excitation fields $h_{1}$ and $h_{2}$. As can be seen from Fig. 8, the amplitude of the component with sum frequency is indeed proportional to the excitation fields. The amplitude of the component increases nearly linearly with $h_{1}$ for a fixed $h_{2}$. It follows from data of Fig. 8 that efficiency of the fields mixing was equal $\alpha_{E}^{m i x}=u\left(f_{ \pm}\right) /\left(b h_{1} h_{2}\right) \approx 30$ $\mathrm{mV} /\left(\mathrm{cm} \cdot \mathrm{Oe}^{2}\right)$. Thus, the efficiency of the fields mixing is approximately equal to the efficiency of the second harmonic generation, which is in agreement with relations (2) and (4).

It is necessary to compare the efficiency of non-resonant processes in the structure with PVDF layers with efficiency of similar processes in structures with other piezoelectric materials. For example, in the PZT-Metglas structure [32], the efficiency of the frequency doubling was $\sim 0.7 \mathrm{~V} /\left(\mathrm{cm} \cdot \mathrm{Oe}^{2}\right)$ at the bending oscillation frequency $f=500 \mathrm{~Hz}$, and the efficiency of the field mixing was $\sim 0.25 \mathrm{~V} /\left(\mathrm{cm} \cdot \mathrm{Oe}^{2}\right)$. Taking into account the acoustic quality factor of bending oscillations $Q \approx 42$, for non-resonant mode we obtain values $\alpha_{E}^{(2)} \approx 17 \mathrm{mV} /\left(\mathrm{cm} \cdot \mathrm{Oe}^{2}\right)$ and $\alpha_{E}^{\text {mix }} \approx 6$ $\mathrm{mV} /\left(\mathrm{cm} \cdot \mathrm{Oe}^{2}\right)$, respectively. It follows, that efficiency of the frequency doubling and magnetic field mixing in the PVDF layer structure turned out to be several times higher than that for a similar structure with piezoceramic layers.

\section{Conclusions}

The direct ME effect of ac magnetic field conversion in ac electrical voltage in the low frequency range $50-1000 \mathrm{~Hz}$ was fully investigated in a flexible planar structure of amorphous ferromagnetic Metglas - piezopolymer PVDF. In linear regime at small amplitudes of the excitation fields the efficiency of non-resonant ME conversion was $\alpha_{\mathrm{E}} \sim 1 \mathrm{~V} /(\mathrm{cm} \cdot \mathrm{Oe})$. The nonlinear $\mathrm{ME}$ effects of the voltage harmonics generation and magnetic fields mixing coincide with the 
increase of amplitudes of the excitation fields. The amplitudes of the harmonics depend nonmonotonically on dc bias magnetic field and have a power dependence on the excitation field. Efficiency of the the $2^{\text {nd }}$ and the $3^{\text {rd }}$ harmonics generation reached $\alpha_{E}^{(2)} \approx 25 \mathrm{mV} /\left(\mathrm{cm} \cdot \mathrm{Oe}^{2}\right)$ and $\alpha_{E}^{(3)} \approx 2.5 \mathrm{mV} /\left(\mathrm{cm} \cdot \mathrm{Oe}^{3}\right)$, respectively. The efficiency of the fields mixing reached $\alpha_{E}^{m i x} \sim 30$ $\mathrm{mV} /\left(\mathrm{cm} \cdot \mathrm{Oe}^{2}\right)$. A simple model that takes into account the non-linear dependence of the magnetostriction of the ferromagnetic layer on the dc magnetic field allows a quantitative explanation of the observed nonlinear phenomena. The efficiency of nonlinear processes is proportional to the derivatives of the magnetostriction over magnetic field. The research conducted here shows that the use of nonlinear ME effects in flexible structures based on PVDF polymer is promising for application in various devices including magnetic field sensors and energy harvesters,

The research was supported by the Ministry of Science and Education of Russian Federation (project № MK-7690.2016.9) and by the Russian Foundation for Basic Research (grant офи_м 162914017\16).

\section{References}

1. L.D. Landau, E.M. Lifshitz, Elektrodinamika sploshnykh sred (Electrodynamics of coutinuous Media), Moscow: Nauka, 1982.

2. C.-W. Nan, M.I. Bichurin, S. Dong, Multiferroic magnetoelectric composites: Historical perspective, status, and future directions, JAP, 103 (2008). 031101.

3. J. Van Suchtelen, Product properties: A new application of composite materials. Philips Res. Rep. 27 (1972) 28.

4. M.M. Vopson, Fundamentals of Multiferroic Meterials and Their Possible applications, Cruitical Reviews in Solid State and Material Sciences, 0 (2014) 1-28.

5. M.I. Bichurin, D.A. Filippov, V.M. Petrov, V.M. Laletin, N. Paddubnaya, G. Srinivasan, Resonance magnetoelectric effects in layered magnetostrictive-piezoelectric composites, Phys. rev. B, 68 (2003) 132408

6. J.F. Scott Applications of Magnetoelectrics, J. Mater. Chem.22 (2012) 4567.

7. H. Palneedi, V. Annapureddy, S. Priya, J. Ryu, Status and perspectives of multiferroic magnetoelectric composite materials and applications, Actuators, 5 (2016) 9.

8. G. Srinivasan, Y.K. Fetisov, Ferrite-piezoelectric layered structures: microwave magnetoelectric effects and electric field tunable devices, Ferroelectrics, 342 (2006) 65.

9. K. Mori, M. Wutting, Magnetoelectric coupluing in Terfenol-D/polyvinyledenefluoride composites, Appl. Phys. Lett. 81 (2002) 100.

10. C.-W. Nan, L. Li, N. Cai, J. Zhai, Y. Ye, H. Lin, L.J. Dong, C.X. Xiong, A three-phase magnetoelectric composites of piezoelectric ceramic, rare-earth iron alloys, and polymer, Appl. Phys. Lett. 81 (2002) 3831.

11. P. Martins, S. Lanceros-Méndez, Polymer-Based Magnetoelectric Materials, Adv. Funct. Mater. 23 (2013) 3371.

12. K.S. Ramadan, D. Sameoto, S. Evoy, A review of piezoelectric polymers as functional matrials for electromechanical transducers, Smart. Meter. Struct. 23 (2014) 033001.

13. N. Cai, J. Zhai, L. Liu, Y. Lin, C.-W. Nan, The magnetoelectric properties of lead zirconate titanate/terfenol-D/PVDF laminate composited, Mater. Science and Eng.,B99 (2003) 211.

14. M. Zeng, S.W. Or, H. Chan, Large magnetoelectric effect from mechanically mediated magnetic field- induced strain effect in Ni-Mn-Ga single crystal and piezoelectric effect in PVDF polymer, J. Alloys and Compounds,490 (2010) 15.

15. M. Silva, S. Reis, C.S. Lehmann, P. Martins, S. Lanceros-Mendes, A. Lasheras, J. Gutierrez, J.M. Barandiran, Optimization of the magnetoelectric responce of poly(vinylidene fluoride)/epoxy/Vitrovac Laminates, Appl. Mater. Interfaces, 5 (2013) 10912.

16. M. P. Silva, P. Martins, A. Lasheras, J. Gutiérrez, J. M. Barandiarán, S. LancerosMendez Size effects on the magnetoelectric response on PVDF/Vitrovac 4040 laminate composites // JMMM. 2015. V. 377. P. 29-33. 
17. J. Gutierrez, A. Lasheras, J.M. Barandiaran, J.L. Vilas, M.S. Sebastian, L.M. Leon, Temperature response of magnetostrictive/piezoelectric polymer magnetoelectric laminates, Key Engineering Materials, 495 (2012) 351.

18. J. Carvell, R. Cheng, P.A. Dowben, Q. Yang, Induced magnetoelectric coupling in ferroelectric/ferromagnetic heteroastructures, APL, 103 (2013) 072902.

19. J Xing, J. Zhai, S. Dong, Z.. Li, and D. Viehland Giant magnetoelectric effect in Metglas/polyvinylidene-fluoride laminates // Appl. Phys. Let. - 2006. V. 89. № 083507.

20. Z. Fang, S.G. Lu, F. Li, S. Datta, Q.M. Zhang, M.E. Tahchi, Enhancing the magnetoelectric response of metglas/polyvinylidene fluoride laminates by exploiting the flux concentration effect // Appl. Phys. Lett. - 2009. V 95. P. 1-3.

21. J. Jin, S.G. Lu, C. Chanthad, Q. Zhang, M.A. Haque, Q. Wang, Multiferroic polymer composites with greatly enhanced magnetoelectric effect under a low magnetic bias // Adv. Mater. - 2011. V. 23. P. 3853-3858.

22. S.G. Lu, J.Z. Jin, X. Zhou, Z. Fzng, Q. Wang, Q.M. Zhang, Large magnetoelectric coupling coefficient in poly(vinilidene fluoride-hexafluoropropylene)/Metglas laminates, J. Appl. Phys. 110 (2011) 104103.

23. J. Li, Y. Li, D. Zhu, Q. Wang, Y. Zhang, Y. Zhu, M. Li, Magnetoelectric effect modulation in a PVDF-Metglas-PZT composite by applying DC electric fields on PZT phase, J. All and comp., 661(2016) 38.

24. A. Kulkarni, K. Meurisch, I. Teliban, R. Jahns, T. Strunskus, A. Piorra, R. Knochel, and E. Faupel, Giant magnetoelectric effect at low frequencies in polymer based thin film composites, APL, 104 (2014) 022904.

25. X.W. Dong, B. Wang, K.F. Wang, J.G. Nan, J.-M. Liu, Ultra-sensitive detection of magnetic field and its direction using bilayer PVDF/Metglas structure, Sensors and Actuators A: Physical, 152 (2009) 64.

26. M-Q Le, F. Behora, A. Comogolub, P.-J. Cottinet, L. Lebrun, A. Hajjaji, Enhenced magnetoelectric effect for flexible current sensor applications, J. Appl. Phys., 115 (2014) 194103.

27. A. Lasheras, J. Gutierrez, S. Reis, D. Sousa, M. Silvar, P. Martins, S. Lanseros-Mendes, J.M. Barandiaran, D.A. Shishkin, A.P. Popov, Energy harvesting device based on a metallic glass/PVDF magnetoelectric laminated composite, Smart Mater. Struct. 24 (2015) 065024.

28. Kamentsev K.E., Fetisov Y.K., Srinivasan G. Low-frequency nonlinear magnetoelectric effects in a ferrite-piezoelectric multilayer, APL, 89 (2006) 142510.

29. L. Y. Fetisov, Y. K. Fetisov, G. Sreenivasulu, and G. Srinivasan Nonlinear resonant magnetoelectric interactions and efficient frequency doubling in a ferromagnetic-ferroelectric layered structure, J. Appl. Phys. 113 (2013) 116101.

30. L.Y. Fetisov, K.E. Kamentsev, G. Srinivasan, Y.K. Fetisov, Frequency dependence of magnetoelectric voltage for a multilayer ferrite-piezoelectric structure with finite conductivity, 106 (2009) 23.

31. Timoshenko S. Vibration Problems in Engineering, D. Van Nostrand, New York, 1961.

32. D. A. Burdin, D. V. Chashin, N. A. Ekonomov, Y. K. Fetisov, L. Y. Fetisov, G. Sreenivasulu, and G. Srinivasan Resonance mixing of alternating current magnetic fields in a multiferroic composite, J. Appl. Phys. 113 (2013) 033902. 